\title{
Aortic Valvuloplasty for Cusp Perforation Caused by Kawasaki Disease
}

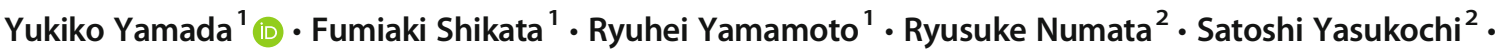 \\ Takamasa Takeuchi ${ }^{1}$
}

Received: 21 October 2020 / Accepted: 22 December 2020 / Published online: 7 January 2021

(C) Dr. K C Chaudhuri Foundation 2021

To the Editor: Kawasaki disease in the acute phase causes cardiac issues including coronary artery lesions (CALs) and valve dysfunction [1]. Kawasaki disease is generally caused by pancarditis and acute hypercytokinemia $[1,2]$. The occurrence of valve insufficiency that required medical treatment is relatively lower than that of CALs recognized as the one of major cardiac complications of Kawasaki disease [1]. Higher moderate aortic regurgitation after Kawasaki disease like that in the present case is rare, and only six surgical cases have been reported to date [3-5], whereas mitral valves are more frequently affected and most mitral regurgitation improves after alleviating the inflammation.

A 7-mo-old patient had high fever for $7 \mathrm{~d}$. After $1 \mathrm{mo}$, she was suspected with Kawasaki disease due to prolonged fever, with the occurrence of polymorphic rash and mucosal change. The diastolic and systolic murmur was noted in the precordial region. A transthoracic echocardiogram (TTE) revealed noncoronary cusp perforation of the aortic valve and severe aortic regurgitation without vegetation. The diameters of left main trunk of the left coronary artery and the proximal right coronary artery were $2.6 \mathrm{~mm}(\mathrm{z}=+3.7)$ and $3.0 \mathrm{~mm}(\mathrm{z}$ $=+6.6$ ), respectively. After a 2 -wk medication, she had no fever without remarkable findings of Kawasaki disease. However, she still showed tachypnea and lethargy. Then, aortic valvuloplasty was planned with Ozaki procedure and Konno surgery in case of severe aortic regurgitation after the repair.

The cardiopulmonary bypass (CPB) was initiated with bicaval drainage and aortic cannulation. After cross-

Yukiko Yamada

yamada.yukiko@twmu.ac.jp

1 Department of Cardiovascular Surgery, Nagano Children's Hospital, 3100 Toyoshina Azumino, Nagano 399-8288, Japan

2 Department of Pediatric Cardiology, Nagano Children's Hospital, Nagano, Japan clamping the ascending aorta, the aortotomy was made. The perforation of $2 \times 3 \mathrm{~mm}$ in size was detected near the annulus of noncoronary cusp. Double interrupted pledgeted stitches using autologous pericardium strips were made to close the leaflet hole. The TEE showed mild aortic regurgitation after $\mathrm{CPB}$ weaning. The postoperative course was free of neurological or respiratory dysfunction. The patient was discharged on the 37 th postoperative day.

Aortic valvuloplasty was successfully performed to repair the aortic cusp perforation after the acute phase of Kawasaki disease. Persistent aortic regurgitation should be carefully followed in the long-term as aortic regurgitation potentially deteriorates.

\section{Compliance with Ethical Standards}

Conflict of Interest None.

\section{References}

1. Tsuda E, Yashiro M, Nakamura Y. Cardiac valvular lesions due to Kawasaki disease: a Japanese nationwide survey. J Pediatr. 2020;218:78-84.

2. Takahashi T, Taniguchi K, Matsuda H. Surgical treatment for aortic regurgitation and left ventricular dysfunction caused by Kawasaki disease. Cardiol Young. 1998;8:123-5.

3. Gidding SS, Shulman ST, Ilbawi M, Crussi F, Duffy CE. Mucocutaneous lymph node syndrome (Kawasaki disease): delayed aortic and mitral insufficiency secondary to active valvulitis. J Am Coll Cardiol. 1986;7:894-7.

4. Fuse S, Tomita H, Ohara T, Iida K, Takamuro M. Severely damaged aortic valve and cardiogenic shock in an infant with Kawasaki disease. Pediatr Int. 2003:45:110-3.

5. Nakano H, Nojima K, Saito A, Ueda K. High incidence of aortic regurgitation following Kawasaki disease. J Pediatr. 1985;107:5963.

Publisher's Note Springer Nature remains neutral with regard to jurisdictional claims in published maps and institutional affiliations. 